\title{
INDICADORES DE DESEMPENHO ESCOLAR E PROBLEMAS DE COMPORTAMENTO NO CONTEXTO DA HOSPITALIZAÇÃO INFANTIL ${ }^{1}$
}

\author{
Mariana Rodrigo do Vale Costa e Silva ${ }^{2}$ \\ https://orcid.org/0000-0003-2822-2724
}

Tayane Costa Marques Martins ${ }^{3}$ https://orcid.org/0000-0001-7897-8779

Tatiane Lebre Dias ${ }^{4}$ https://orcid.org/0000-0002-9515-1578

RESUMO: O presente artigo de natureza quanti-qualitativa analisou o desempenho escolar e problemas de comportamento de crianças hospitalizadas. Participaram seis crianças (média de idade $=6,5$ ) hospitalizadas em hospital público de Cuiabá-MT, com doença aguda e crônica. No estudo foi utilizado a Lista de Verificação Comportamental para crianças/adolescentes de 6/18 anos (CBCL), Instrumento de Avaliação de Repertório Básico para a Alfabetização (IAR) e a Escala de Avaliação de Estratégias de Aprendizagem para o Ensino Fundamental (EAVAP-EF). Os resultados apontaram que todas as crianças apresentaram resultado abaixo da média em estratégias de aprendizagem, a maioria indicou dificuldade na alfabetização. Na CBCL três crianças obtiveram classificação de perfil clínico nas escalas de competência e em problemas de comportamento internalizantes. De modo geral, observou-se dificuldades nas áreas avaliadas, demonstrando a necessidade de programas de intervenção psicoeducativos para crianças hospitalizadas.

PALAVRAS CHAVE: Hospitalização, Criança, Comportamento, Aprendizagem.

10 presente artigo é resultado de projeto de pesquisa que contou com financiamento da Fundação de Amparo à Pesquisa do Estado de Mato Grosso - FAPEMAT (Processo nr. 214901/2015), com Bolsa de Iniciação Científica para a segunda autora.

2 Psicóloga pela UFMT. Mestranda do Programa de Pós-Graduação em Psicologia da Universidade Federal de Mato Grosso (UFMT). Cuiabá/MT - Brasil.marianarvcs@gmail.com

3 Psicóloga pela UFMT. Especialista em Terapia Cognitivo Comportamental. Bolsista de Iniciação Científica do projeto de pesquisa que contou com financiamento da FAPEMAT Processo nr. 214901/2015). Cuiabá/ MT - Brasil. tayanecostamarques@gmail.com

4 Doutora em Psicologia. Docente do Departamento de Psicologia da UFMT e do Programa de Pós-Graduação em Psicologia da UFMT. tatianelebre@gmail.com

Rev. Fac. Educ. (Univ. do Estado de Mato Grosso), Vol. 35, Ano 20, № 1, p. 35-50, jan./jun, 2021 (Epub Ahead of Print 12 maio, 2021) 


\section{INDICATORS OF SCHOOL PERFORMANCE AND BEHAVIOR PROBLEMS IN THE CONTEXT OF CHILD HOSPITALIZATION}

ABSTRACT: The present article of a quantitative and qualitative nature analyzed the school performance and behavior problems of hospitalized children. Six children (mean age $=6.5$ ) hospitalized in a public hospital in Cuiabá-MT, with acute and chronic illness, participated. In the study, the Behavioral Checklist for children / adolescents aged 6/18 (CBCL), the Basic Literacy Assessment Tool (IAR) and the Learning Strategies Assessment Scale for Elementary Education (EAVAP-) were used. EF). The results showed that all children presented below average results in learning strategies, the majority indicated difficulties in literacy. In the $\mathrm{CBCL}$, three children obtained a classification of clinical profile in the competence scales and in internalizing behavior problems. In general, difficulties were observed in the areas evaluated, demonstrating the need for psychoeducational intervention programs for hospitalized children.

KEY WORDS: Hospitalization, Child, Behavior, Learning

\section{INDICADORES DE DESEMPEÑO ESCOLAR Y PROBLEMAS DE COMPORTAMIENTO EN EL CONTEXTO DE HOSPITALIZACIÓN INFANTIL}

RESUMEN: El presente artículo de carácter cuanti-cualitativo analizó el desempeño escolar y los problemas de conducta de los niños hospitalizados. Participaron seis niños (edad media $=6,5$ ) hospitalizados en un hospital público de Cuiabá-MT, con enfermedad aguda y crónica. En el estudio, se utilizó la Lista de Verificación de Comportamiento para niños / adolescentes de 6/18 años (CBCL), la Herramienta de Evaluación de Alfabetización Básica (IAR) y la Escala de Evaluación de Estrategias de Aprendizaje para Educación Primaria (EAVAP-). EF). Los resultados mostraron que todos los niños presentaron resultados por debajo del promedio en las estrategias de aprendizaje, la mayoría indicó dificultades en la alfabetización. En $\mathrm{CBCL}$, tres niños obtuvieron clasificación de perfil clínico en las escalas de competencia y en la internalización de problemas de conducta. En general, se observaron dificultades en las áreas evaluadas, evidenciando la necesidad de programas de intervención psicoeducativa para niños hospitalizados.

PALABRAS CLAVES: Hospitalización, Niño, Comportamiento, Aprendizaje.

\section{Introdução}

No percurso do desenvolvimento da criança é esperado que o mesmo ocorra de forma equilibrada, entretanto, o surgimento de uma doença pode 
causar situações inesperadas para a própria criança e a família. Nesse caso em se tratando de doença crônica, ou seja, doença que apresenta “... início gradual, com duração longa ou incerta (...) múltiplas causas, e o tratamento envolve mudanças de estilo de vida, em um processo de cuidado contínuo que usualmente não leva à cura" (CONASS, 2012, p. 2), pode ocasionar diversas consequências como recorrentes hospitalizações, busca por diagnóstico e tratamento. De acordo com dados do Ministério da Saúde entre os anos de 2015 e 2106 a média de permanência hospitalar de crianças e adolescentes no país foi de 5,8 dias e, em Mato Grosso a média foi de 5,2 dias (MS, 2016).

O processo de hospitalização da criança e do adolescente pode gerar uma séria de adventos como agravamento do estresse em função de não conseguir compreender o que está acontecendo em seu organismo (SILVEIRA et al, 2018, 2019), restrição das atividades (LIMA \& SANTOS, 2015), a ruptura no cotidiano, afastamento da moradia, escola, amigos, familiares, a criança precisa se adaptar a rotina hospitalar, a permanência no leito por um tempo maior que o habitual, a alimentação diferenciada, diminuição ou proibição do brincar (JANSEN et al, 2010; FARIAS et al, 2017) e as consequências para a família de como vivencia esse processo (GOMES et al, 2016). Outras situações estressoras para as crianças hospitalizadas são os tratamentos invasivos, a necessidade de ser medicada e a falta de controle da situação que está vivendo (FARIAS, 2019; MENÇA e SOUSA, 2013). No hospital, de acordo com Barros (1998, p. 13) são inúmeras as situações causadoras de estresse, dentre elas destacam-se "... a presença de equipamento estranho e ameaçador, a necessidade de administrar tratamentos ou meios de diagnóstico assustadores e dolorosos".

Nessa perspectiva Ribeiro e Junior (2009) ao realizarem um estudo com crianças de 7 a 12 anos utilizando o método de desenho e estória com o tema do hospital verificaram a existência de dois princípios distintos de compreensão em relação à "hospitalização/hospital", o primeiro tinha uma visão de hospital como um lugar de ajuda, tratamento, apoio e salvação, entretanto, enquanto o segundo era percebido como um local de privação, sofrimento, punição e castigo. A esse respeito Xavier (2012) salienta que processo de adoecimento, seja agudo ou crônico, pode estar ligado a diversos fatores, como a predisposição genética, qualidade de vida, violência, entre outros aspectos. Desse modo Simonetti (2004, p. 71) considera que "tanto o inesperado de uma doença aguda como o prolongamento de uma doença crônica gera aspectos psicológicos bem distintos". Também Castro e Piccinini (2002) salientam o impacto da doença crônica na capacidade funcional da criança, e a necessidade de internação devido à condição ser mais recorrente. 
Por consequência da doença e da hospitalização, a criança se afasta da família e da escola, porém, seus direitos foram garantidos a partir da Lei no 8.069 de 1990 (BRASIL, 1990) que institui o Estatuto da Criança e do Adolescente (ECA) e pela Resolução no 41 de 1995 do Conselho Nacional dos Direitos da Criança e do Adolescente (CONANDA) que institui os direitos das crianças e dos adolescentes hospitalizados com direitos como proteção à vida e a saúde; ser hospitalizado quando necessário; ser acompanhados por um responsável; receber visita; desfrutar de alguma forma de recreação, programas de educação para a saúde, acompanhamento do currículo escolar durante sua permanência hospitalar (BRASIL, 1995).

A escolarização das crianças com doenças crônicas torna-se mais complicada por causa das internações de longo prazo, podendo chegar a meses. Na tentativa da redução dos danos causados pela ausência na sala de aula de crianças e adolescentes em hospitalização, a Resolução CNE/CEB no 02/2001, que institui diretrizes nacionais para a educação especial na educação básica, garante educação aos alunos que estão impossibilitados de frequentar as aulas devido a tratamento hospitalar.

Assim a classe hospitalar tem como propósito favorecer a construção do conhecimento no âmbito da educação básica, respeitando as capacidades e necessidades especiais de cada indivíduo, seja ele criança, adolescente ou adulto (MINISTÉRIO DA EDUCAÇÃO, 2001). A criação desse espaço é uma conquista no âmbito da educação especial, garantindo que o acompanhamento pedagógico-educacional não seja comprometido pelos processos de internação. De acordo com a Secretaria de Educação Especial (BRASIL, 2002, p. 13) a classe hospitalar refere-se a "atendimento pedagógico-educacional que ocorre em ambientes de tratamento de saúde, seja na circunstância de internação[...] atendimento em hospital-dia e hospital-semana ou em serviços de atenção integral à saúde mental".

O reconhecimento de que a criança pode necessitar de apoio educacional em diferentes situações requer a necessidade de projetos que disponibilizem recursos à criança hospitalizada, um atendimento pedagógico capaz de fazer com que a criança não se sinta incapaz e impossibilitada de realizar atividades. Desse modo o atendimento pedagógico realizado no ambiente hospitalar deverá se concretizar com atividades que envolvam o processo de desenvolvimento físico, cognitivo e emocional, para que estes não se interrompam frente à doença, mais sim, que ganhe novas perspectivas frente à realidade atual da criança (CALEGARI-FALCO, 2007; MENZANI et al, 2017). Dessa forma, o trabalho a ser realizado, com os alunos hospitalizados, deve sempre ser de 
maneira lúdica e com diálogo com o intuito de junto com a criança buscar compreender melhor a realidade que a cerca.

Em uma investigação Hostert et al (2014) verificaram presença de comportamentos como dor de cabeça e medo em crianças com câncer que frequentavam a classe hospitalar. De acordo com os autores o desenvolvimento de atividades lúdicas e assistência psicológica podem auxiliar a criança no enfrentamento da doença. Em outro estudo que investigou as estratégias de enfrentamento da hospitalização infantil Moraes e Enumo (2008) ao utilizarem instrumentos informatizado específico observaram maior frequência de comportamentos facilitadores para a hospitalização, enquanto a ruminação e distração foram a estratégias de enfrentamento mais utilizadas. Também verificaram indicação de problemas de comportamento, considerando a necessidade de suporte para as crianças em situação de internação.

Diante desse contexto o estudo investigou o desempenho escolar, as estratégias de aprendizagem e problemas de comportamento de crianças hospitalizadas.

\section{Método}

O estudo se caracteriza por uma abordagem quanti-qualitativa, no qual participaram seis crianças (dois meninos e quatro meninas) internadas em uma instituição de saúde da pública da cidade de Cuiabá/MT, que serão identificadas pela letra " $C$ " seguida de número. Os critérios de inclusão incluíram hospitalização da criança no mínimo de 48 horas e motivo de internação por condição aguda ou crônica. O critério de exclusão foi não aceitação dos pais/responsáveis em não participar da pesquisa.

Os instrumentos utilizados foram:

a) Instrumento de Avaliação de Repertório Básico para a Alfabetização - IAR, desenvolvido por Leite (1984), o qual tem como objetivo avaliar o repertório comportamental das crianças acerca dos pré-requisitos necessários para a aprendizagem da leitura e da escrita, fornecendo informações sobre as condições iniciais para o início da alfabetização e fornecer aos professores informações sobre as habilidades e conceitos que precisam ser trabalhados para que a criança aprenda processos de leitura e escrita;

b) Escala de Avaliação de Estratégias de Aprendizagem para o Ensino Fundamental - EAVAP-EF, de Oliveira et al (2010) avalia as estratégias de aprendizagem relatadas e empregadas por alunos do Ensino Fundamental, identificando de forma específica as estratégias cognitivas e metacognitivas 
e ausência ou não de estratégias metacognitivas disfuncionais, sendo este destinado para uma população com idades entre sete e dezesseis anos matriculados no Ensino Fundamental. Sua aplicação pode ser feita coletiva ou individualmente;

c) Child Behavior CheckList - Lista de Verificação Comportamental para crianças/adolescentes de 6/18 anos CBCL, de Achenbach e Rescorla (2010) é utilizado para avaliação de problemas de comportamento, contém 138 itens, sendo 20 referentes à avaliação de aptidão social e 118 referentes a problemas de comportamento de crianças com idades entre seis e 18 anos. Nessa lista, as questões são respondidas pelos pais e/ou cuidadores, que respondem conforme suas concepções de sua criança contrastando com o comportamento de outra criança com mesma idade.

A coleta de dados foi realizada no ano de 2016, de forma individualizada, na brinquedoteca da enfermaria pediátrica, com duração média de 40 minutos. O projeto de pesquisa foi aprovado pelo Comitê de Ética em Pesquisa HUJM/UFMT (Parecer no 1083963/2015). Para a coleta os pesquisadores receberam treinamento em relação à aplicação dos instrumentos. $O$ primeiro contato foi realizado com o pai/responsável com o objetivo de explicar a pesquisa e apresentar o Consentimento Livre e Esclarecido (CLE), somente após a assinatura pelo responsável foi realizado o contato com a criança explicando o objetivo da pesquisa e solicitado assinatura do Termo de Assentimento (TA). No caso da criança não ser alfabetizada foi utilizada a assinatura por meio do dedo polegar.

Após a coleta os instrumentos foram corrigidos de acordo com os manuais de correção e interpretação. No IAR foi analisada a proporção de acertos nos testes de modo a se obter o total, permitindo ao final uma classificação em "muita dificuldade" (M) ou "alguma dificuldade" (A). Na EAVAP-EF a partir dos dados obtidos no crivo de correção, tem-se o resultado individual que permite encontrar a média obtida pela criança e a classificação em: Ausência de estratégias metacognitivas disfuncionais (A); Estratégias Cognitivas (C) e Estratégias Metacognitivas (M). Os dados da $\mathrm{CBCL}$ foram analisados de acordo com a instrução, obtendo ao final classificação para as competências (atividade, social, escola) e problemas de comportamento (internalizantes e externalizantes) em clínico, limítrofe ou normal. 


\section{Resultados e discussão}

Os participantes tinham idade entre 6 e 7 anos $(M=6,5 ; D P=0,547)$, com diferentes situações em relação ao processo de adoecimento e condição de saúde. A C1, com seis anos de idade e diagnóstico de fenilcetonúria, doença ligada à deficiência da enzima fenilalanina hidroxilase. Segundo Mira e Marques (2000) "[...] pacientes com PKU clássica apresentam deficiência na pigmentação (cabelos e pele claros) devido à inibição completa da hidroxilação da tirosina pela tirosinase. Já C2, com seis anos, com diagnóstico de mucopolissacarídeos II, também conhecida como Síndrome de Hunter, faz parte de um grupo de doenças causadas pela deficiência de enzimas lisossômicas. Trata-se de doença genética cujas manifestações clínicas mais frequentes estão ligadas a alterações na estrutura corporal do afetado, causando baixa estatura, contraturas articulares, face grosseira e alterações esqueléticas, e atraso no desenvolvimento psicomotor, infecções nas vias aéreas superiores e inferiores, surdez e cardiopatia (PINTO et al, 2006).

As crianças C3 e C4, com 7 anos de idade e diagnóstico de Diabetes Mellitus tipo 1, que se caracteriza por uma doença metabólica por hiperglicemia. De acordo com Marcelino et al (2005, p. 72), apresenta entre os principais sintomas "muita sede (polidipsia), excesso de urina (poliúria), muita fome (polifagia), e emagrecimento". Também se fazem presentes "sonolência, dores generalizadas, formigamentos e dormências, cansaço doloroso nas pernas, câimbras, nervosismo, indisposição para o trabalho, desânimo, turvação da visão, cansaço físico e mental" (MARCELINO et al, 2005, p. 72). De acordo com autores, podem surgir complicações em caso de problemas no controle da doença na área da visão (retinopatia), nos rins (nefropatia) e problemas neurológicos (neuropatia). Até o momento da pesquisa a C5 e C6, ambas com seis anos de idade, encontravam-se em investigação diagnóstico.

Em relação à aprendizagem, do total de crianças investigadas quatro $(\mathrm{C} 1, \mathrm{C} 2, \mathrm{C} 5, \mathrm{C} 6)$ encontrava-se em processo de alfabetização, enquanto duas $(\mathrm{C} 3, \mathrm{C} 4)$ já eram alfabetizadas. Desse modo, o primeiro grupo de crianças foi avaliado utilizando o Instrumento de Avaliação de Repertório Básico para a Alfabetização (IAR) e o segundo grupo utilizando a Escala de Avaliação de Estratégias de Aprendizagem para o Ensino Fundamental (EAVAP-EF).

No IAR, as crianças apresentaram resultados satisfatórios, sendo que três crianças foram classificadas como tendo alguma dificuldade $(C 2, C 5, C)$ e uma delas como tendo muita dificuldade (C1). Duas crianças (C1 e C2) não conseguiram completar o instrumento sob alegação de cansaço (Tabela 1). 
Tabela 1. Resultados no Instrumento de Avaliação de Repertório Básico para a Alfabetização - IAR ( $n=4)$

\begin{tabular}{|c|c|c|c|c|c|c|c|c|c|}
\hline Área & C1 & C2 & C5 & C6 & Área & C1 & C2 & C5 & C5 \\
\hline $\begin{array}{c}\text { I Esquema } \\
\text { Corporal }\end{array}$ & 0,83 & 0,83 & 1 & 1 & VIII Forma & 0 & 1 & 1 & 0,75 \\
\hline $\begin{array}{c}\text { II } \\
\text { Lateralidade }\end{array}$ & 0,5 & 1 & 1 & 1 & $\begin{array}{c}\text { IX } \\
\begin{array}{c}\text { Discriminação } \\
\text { Visual }\end{array}\end{array}$ & 0,5 & 0,33 & 0,83 & 0,83 \\
\hline III Posição & 0,5 & 1 & 1 & 1 & $\begin{array}{c}X \\
\text { Discriminação } \\
\text { Auditiva }\end{array}$ & 0 & - & 0,75 & 1 \\
\hline IV Direção & 1 & 1 & 1 & 1 & $\begin{array}{c}\text { XI } \\
\text { Verbalização } \\
\text { da Palavra }\end{array}$ & - & - & - & - \\
\hline V Espaço & 1 & 0,5 & 1 & 1 & $\begin{array}{l}\text { XII Análise- } \\
\text { Síntese }\end{array}$ & - & - & 0,33 & 0,66 \\
\hline VI Tamanho & 0,75 & 0,88 & 1 & 1 & $\begin{array}{l}\text { XIII Coord. } \\
\text { Motora Fina }\end{array}$ & - & - & - & 1 \\
\hline \multirow[t]{2}{*}{$\begin{array}{c}\text { VII } \\
\text { Quantidade }\end{array}$} & 1 & 1 & 1 & 1 & Média final & 0,46 & 0,58 & 0,76 & 0,86 \\
\hline & & & & & Classificação & M & $A$ & $A$ & $A$ \\
\hline
\end{tabular}

Legenda:

M: Muita dificuldade;

A: Alguma dificuldade.

De acordo com a Tabela 1 verifica-se de modo geral presença de dificuldade de aprendizagem para áreas avaliadas pelo IAR. Em estudo que utilizou o mesmo instrumento Oliveira et al (2011) ao avaliar o desempenho em crianças prematuras e nascidas a termo, observou que o primeiro grupo apresentou dificuldades significativamente maior que o do segundo grupo. $\mathrm{Na}$ perspectiva do professor Fontes (2005) numa abordagem qualitativa de investigação observou que assim como as possibilidades de atuação do professor nesse espaço são grandes, do mesmo modo são os desafios de uma prática educativa no campo hospitalar. Recentemente Belancieri (2018) ao proporem uma intervenção em pacientes pediátricos com contação de histórias observaram que essa estratégia facilita o processo de hospitalização e aproximação com o cotidiano escolar.

Na Escala de Avaliação de Estratégias de Aprendizagem para o Ensino Fundamental (EAVAP-EF) as crianças apresentaram desempenho abaixo da 
média estabelecida para a idade $(C 3=33, C 4=34)$. Apesar do resultado da média geral ser próximo, as pontuações por subescalas de C3 e C4 mostraram desempenhos diferenciados, de modo que C3 pontuou mais na subescala Estratégias Cognitivas e C4 na subescala Ausência de Estratégias Metacognitivas Disfuncionais (Tabela 2).

Tabela 2. Média de acertos na Escala de Avaliação de Estratégias de Aprendizagem para o Ensino Fundamental - EAVAP-EF ( $N=2)$

\begin{tabular}{c|cccc}
\hline Crianças & A & C & M & Média Geral \\
\hline C3 & 8 & 16 & 9 & 33 \\
C4 & 26 & 5 & 3 & 34 \\
\hline Média 7-10 anos pela Escala & 18,7 & 10,6 & 8,7 & 38 \\
\hline
\end{tabular}

Legenda:

A: Ausência de estratégias metacognitivas disfuncionais;

C: Estratégias Cognitivas;

M: Estratégias Metacognitivas

Em investigação que avaliou as estratégias de aprendizagem e a relação com o desempenho escolar em crianças com queixa de aprendizagem escolar do 20 ano do Ensino Fudamental, Prates et al (2016) verificaram correlação significativa entre ausência de estratégias metacognitivas disfuncionais, estratégias cognitivas, estratégias metacognitivas e o desempenho escolar em escrita, aritmética, leitura e total. Neste estudo não se pode afirmar que o desempenho das crianças foi afetado pela doença ou hospitalizações, entretanto, verificou-se dificuldades nas estratégias avaliadas demonstrando a necessidade de intervenção nessas áreas.

Em relação aos problemas de comportamento, do total de crianças avaliadas três delas (C2, C3, C4) apresentaram indicação de problemas com perfil "clínico" para problemas internalizantes, ou seja, sugestão de comportamentos de ansiedade, depressão, retraimento. Das três crianças duas delas (C2 e C6) também obtiveram perfil clínico para indicação de problemas externalizantes como comportamento agressivo, problemas de atenção, de pensamentos e sociais (Tabela 3). Lorencini e Paula (2015) verificaram resultado semelhante com maior pontuação para problemas internalizantes num grupo de 12 crianças com diagnóstico de doença falciforme. 
Em estudo de metanálise Pinquart e Shen (2011) revisaram estudos que aplicaram a Child Behavior Check List (CBCL), a Youth Self Report (YSR) e o Teacher Report Form (TRF) em crianças e adolescentes com doença crônica física e com risco de problemas emocionais e comportamentais. Os autores verificaram altos níveis de problemas internalizantes (ansiedade, depressão, por exemplo), problemas externalizantes e problemas comportamentais nas crianças mais novas com doença crônica do que nos pares saudáveis. Os autores chamam a atenção para os aspectos que se referem ao sofrimento psicológico e a necessidade de encaminhamentos para os serviços de saúde mental nessa população.

Tabela 3. Resultados das crianças em relação aos problemas de comportamento no Children Behavior Check List (CBCL) ( $\mathrm{N}=6)$

\begin{tabular}{c|cccccc}
\hline Criança/CBCL & C1 & C2 & C3 & C4 & C5 & C6 \\
\hline Problemas internalizantes & - & - & - & - & - & - \\
Ansiedade/ Depressão & $\mathrm{N}$ & $\mathrm{C}$ & $\mathrm{C}$ & $\mathrm{N}$ & $\mathrm{N}$ & $\mathrm{N}$ \\
\hline Queixas somáticas & $\mathrm{N}$ & $\mathrm{N}$ & $\mathrm{N}$ & $\mathrm{N}$ & $\mathrm{N}$ & $\mathrm{N}$ \\
\hline Retraimento & $\mathrm{N}$ & $\mathrm{C}$ & $\mathrm{N}$ & $\mathrm{N}$ & $\mathrm{N}$ & $\mathrm{N}$ \\
\hline Total & $\mathrm{N}$ & $\mathrm{C}$ & $\mathrm{C}$ & $\mathrm{C}$ & $\mathrm{N}$ & $\mathrm{N}$ \\
\hline Problemas externalizantes & - & - & - & - & - & - \\
\hline Problemas sociais & $\mathrm{N}$ & $\mathrm{C}$ & $\mathrm{N}$ & $\mathrm{N}$ & $\mathrm{N}$ & $\mathrm{N}$ \\
\hline $\begin{array}{c}\text { Problemas de pensamento } \\
\text { Problemas de atenção }\end{array}$ & $\mathrm{N}$ & $\mathrm{C}$ & $\mathrm{N}$ & $\mathrm{N}$ & $\mathrm{N}$ & $\mathrm{N}$ \\
\hline Comportamento agressivo & $\mathrm{N}$ & $\mathrm{C}$ & $\mathrm{N}$ & $\mathrm{N}$ & $\mathrm{N}$ & $\mathrm{N}$ \\
\hline $\begin{array}{c}\text { Tegenda: } \\
\text { Compotal }=\text { Clínico. }\end{array}$ & $\mathrm{N}$ & $\mathrm{N}$ & $\mathrm{N}$ & $\mathrm{N}$ & $\mathrm{N}$ & $\mathrm{N}$ \\
\hline
\end{tabular}


No que se refere às competências avaliadas pelo $\mathrm{CBCL}$, apenas uma criança apresentou perfil clínico para a competência escolar e uma para competência atividade. No perfil geral três crianças $(C 2, C 3, C 4)$ indicaram perfil clínico, sendo que uma criança $(\mathrm{C} 1)$ não obteve resultado, pois não apresentou respostas para a competência escolar, afetando o resultado final (Tabela 4).

Tabela 4. Resultado das crianças nas escalas de competência no Child Behavior Check List $(\mathrm{CBCL})(\mathrm{N}=6)$

\begin{tabular}{|c|c|c|c|c|}
\hline \multirow[t]{2}{*}{ Criança } & \multirow[t]{2}{*}{ Perfil Geral } & \multicolumn{3}{|c|}{ Competências } \\
\hline & & Atividade & Social & Escola \\
\hline $\mathrm{C} 1$ & NR & $\mathrm{N}$ & $\mathrm{N}$ & NR \\
\hline $\mathrm{C} 2$ & C & $\mathrm{N}$ & $\mathrm{N}$ & C \\
\hline $\mathrm{C} 3$ & C & C & $\mathrm{N}$ & $\mathrm{N}$ \\
\hline $\mathrm{C} 4$ & C & $\mathrm{N}$ & $\mathrm{N}$ & $\mathrm{N}$ \\
\hline $\mathrm{C} 5$ & $\mathrm{~N}$ & $\mathrm{~N}$ & $\mathrm{~N}$ & $\mathrm{~N}$ \\
\hline $\mathrm{C} 6$ & $\mathrm{~N}$ & $\mathrm{~N}$ & $\mathrm{~N}$ & $\mathrm{~N}$ \\
\hline
\end{tabular}

Legenda:

$\mathrm{NR}=$ Não respondeu;

$\mathrm{N}=$ Normal;

C= Clínico

A indicação de problemas de comportamento nas crianças avaliadas assemelha-se aos resultados encontrados por Moraes e Enumo (2008) com crianças de idades entre seis e doze anos hospitalizadas entre cinco e vinte 
dias. No estudo prevaleceu perfil normal em relação à competência social, diferentemente dos resultados encontrados por Lorencini e Paula (2015) em que $83 \%$ das crianças obtiveram nível clínico nos itens "competência social". Ao analisaram três áreas de funcionamento - social, acadêmico e físico - de crianças com doença crônica Pinquart e Teubert (2011) verificaram maior diminuição no funcionamento físico do que o acadêmico e o social.

\section{Considerações finais}

O tratamento de saúde da criança hospitalizada vai além dos aspectos biológicos, pois a experiência de adoecimento e internação implica mudanças na rotina, distanciamento de familiares, dos amigos, de objetos significativos, da escola, além dos procedimentos invasivos e dolorosos. Com isso, é relevante assegurar direitos básicos como o acesso ao lazer, ao convívio no meio externo, às informações e ao exercício intelectual.

A classe hospitalar objetiva atender às crianças em relação aos aspectos da aprendizagem que podem ser afetados pelas condições de saúde. Desse modo é fundamental a presença de classe hospitalar visando minimizar os efeitos da doença e da própria hospitalização e garantir os direitos à educação.

O estudo investigou aspectos da aprendizagem e indicação de problemas de comportamento em crianças no contexto da hospitalização. Os resultados revelaram dificuldades nas áreas avaliadas, entretanto, não se pode afirmar que esses resultados estão relacionados à doença ou hospitalização. Diante disso, observa-se a necessidade de outras investigações considerando a limitação em relação ao número de participantes. Outros fatores significativos podem contribuir para esse resultado como apoio e suporte familiar, interação com a equipe de saúde, método de ensino, tempo de internação, evolução da condição clínica.

Assim espera-se por mais pesquisas que abordem aspectos da educação no contexto da hospitalização da criança, assim como fatores socioemocionais que permitam melhorar a qualidade de vida da criança nesse contexto e a proposição de programas de intervenção direcionados.

\section{Referências}

ACHENBACH, T. M.; RESCORLA, L. A. Mental health practitioners' guide for the Achenbach system of empirically based assessment (ASEBA). 7th ed. Burlington: University of Vermont, Research Center for Children, Youth, \& Families, 2010. 
BARROS, L. As consequências psicológicas da hospitalização infantil: prevenção e controle. Análise Psicológica, v.1, n. 16, p. 11-28, 1998.

BELANCIERI, M. F.; RODRIGUES, K. R.; CAPELLINI, V. L. M. F.; REIS, V. L. Pedagogia hospitalar: intervenções na unidade pediátrica a partir da contação de histórias. Semin., Ciênc. Soc. Hum., v. 39, n. 1, p. 53-64, 2018.

BRASIL. Ministério da Saúde. Secretaria de Assistência à Saúde Programa Nacional de Humanização da Assistência Hospitalar / Ministério da Saúde, Secretaria de Assistência à Saúde. - Brasília: Ministério da Saúde, 2001.

BRASIL. Resolução CNE/CEB no 02, de 11 de setembro de 2001. Institui as Diretrizes Nacionais para a Educação Especial na Educação Básica. Brasília, DF, 2001.

BRASIL. Secretaria de Educação Especial. Ministério da Educação. Classe hospitalar e atendimento pedagógico domiciliar: estratégias e orientações. Brasília: MEC, SEEP, 2002. Disponível em: http://portal.mec.gov.br/seesp/arquivos/pdf/livro9.pdf.

BRASIL. Lei no. 8029 de 2990. Dispões sobre o Estatuto da Criança e do Adolescente e dá outras providências. Disponível em: http://www.planalto.gov. br/ccivil_03/leis/l8069.htm

BRASIL. Conselho Nacional dos Direitos da Criança e do Adolescente. Resolução $n^{\circ} 41$ de Outubro de 1995.

CALEGARI-FALCO, A. M. Classe Hospitalar: a criança no centro do processo educativo. VII Congresso Nacional de Educação - EDUCERE, Paraná, nov. 2007, p. 4282-4291. Disponível em: <http://www.pucpr.br/eventos/educere/ educere2007/anaisEvento/arquivos/PA-543-12.pdf>

CASTRO, E. K.; PICCININI, C. A. Implicações da doença orgânica crônica na infância para as relações familiares: algumas questões teóricas. Psicol. Reflex. Crit. v. 15, n. 3, p. 625-635, 2002. Disponível em: <http://www.scielo.br/scielo.php?script=sci_arttext\&pid=S0102-79722002000300016\&Ing=en\&nrm=iso>.

CONSELHO NACIONAL DE SECRETÁRIOS DE SAÚDE (CONASS). Nota técnica. Conass 30 anos - Promovendo saúde para todos os brasileiros. 2012. Disponível em: http://www.conass.org.br/biblioteca/wp-content/uploads/2012/02/ NT-41_2012-Rede-Cronicas-final.pdf.

FARIAS, D. D., GABATZ, R. I. B., TERRA, A. P., COUTO, G. R., MILBRAYH, V. M., SCHWARTZ, E. A hospitalização na perspectiva da criança: uma revisão inte-

Rev. Fac. Educ. (Univ. do Estado de Mato Grosso), Vol. 35, Ano 20, № 1, p. 35-50, jan./jun, 2021 (Epub Ahead of Print 12 maio, 2021 ) 
grativa. Rev Enferm UFPE, v. 11, n. 2, p. 703-11, 2017.

FARIAS, D., BÄRTSCHIGABATZ, R. I., MILBRATH, V. M., SCHWARTZ, E.; FREITAG, V. L. Percepção infantil sobre a necessidade de hospitalização para o reestabelecimento da saúde. Revista Enfermagem Atual In Derme, v, 87, n, 25, p. 1-7, 2019.

FONTES, R. S. A escuta pedagógica à criança hospitalizada: discutindo o papel da educação no hospital. Rev. Bras. Educ, n.29, p.119-138, 2005. Disponível em: <http://www.scielo.br/scielo.php?script=sci_arttext\&pi$\mathrm{d}=\mathrm{S} 1413-24782005000200010 \&$ Ing=en\&nrm=iso >.

GOMES, G. C.; ERDMANN, A. L.; OLIVEIRA, P. K.; XAVIER, D. M.; SANTOS, S. S. C.; FARIAS, D. H. R. A família durante a internação hospitalar da criança: contribuições para a enfermagem. Esc Anna Nery, V. 18, N. 2, p. 234-240, 2014. 2014.

GOMES, G. L., FERNANDES, M. G. M., NÓBREGA, M. M. L. Ansiedade da hospitalização em crianças: análise conceitual. Rev Bras Enferm (Internet), v. 69, n. 5, p. 940-945, 2016.

HOSTERT, P. C. C. P.; ENUMO, S. R. F. LOSS, A. B. M. O brincar e problemas de comportamento de crianças com câncer em classes hospitalares. Revista Psicologia: Teoria e Prática, v, 16, n. 1, p. 127-140, 2014.

JANSEN, M. F.; SANTOS, R. M.; FAVERO, L. Benefícios da utilização do brinquedo durante o cuidado de enfermagem prestado à criança hospitalizada. Rev Gaúcha Enferm. v. 31, n. 2, p. 247-253, 2010.

LEITE, A. A. S. Inventário de avaliação do repertório básico para a alfabetização. São Paulo: Edicon, 1984.

LIMA, K. Y. N.; SANTOS, V. E. P. O lúdico como estratégia no cuidado à criança com câncer. Revista Gaúcha de Enfermagem, 36(2), p. 76-81, 2015. https:// doi.org/10.1590/1983-1447.2015.02.51514

LORENCINI, G. R.F.; PAULA, K. M. P. Perfil comportamental de crianças com anemia falciforme. Temas Psicol. [online], v. 23, n. 2, p. 269-280, 2015. http:// dx.doi.org/10.9788/TP2015.2-03.

MENÇA, V. B.; SOUSA, S. S. P. S. A criança e o processo de hospitalização: os desafios promovidos pela situação da doença. Psicodom - Universidade Dom Bosco, Porto Alegre, 11 ed, jul., 2013.

MENZANI, R. M.; REGUEIRO, E. M. G.; DE CASSIA L. J. Ser criança na classe 
hospitalar: a dimensão psicológica na interface educação e saúde. Revista Brasileira Multidisciplinar, São Paulo, v. 20, n. 1, p. 106-120, 2017. Disponível em: <https://doi.org/10.25061/2527-2675/ReBraM/2017.v20i1.476>.

MINISTÉRIO DA SAÚDE. Sistema de Informações Hospitalares do SUS (SIH/ SUS). Morbidade Hospitalar do SUS. 2016. Disponível em: http://tabnet.datasus.gov.br/cgi/tabcgi.exe?sih/cnv/niuf.def

MORAES, E. O.; ENUMO, S. R. F. Estratégias de enfrentamento da hospitalização em crianças avaliadas por instrumento informatizado. PsicoUSF, v. 13, n. 2, p. 221-231, 2008. Disponível em: <http://pepsic.bvsalud.org/scielo. php? script=sci_arttext\&pid=S1413-82712008000200009\&lng=pt\&nrm=iso $>$.

OLIVEIRA, C. G. T. et al. Indicadores cognitivos, linguísticos, comportamentais e acadêmicos de pré-escolares nascidos pré-termo e a termo. Psicologia: Teoria e Pesquisa, v. 27, n. 3, p. 283-290, 2011.

OLIVEIRA, K. L; BORUCHOVITCH, E \& SANTOS, A. A. A. Escala de avaliação das estratégias de aprendizagem para o ensino fundamental: EAVAP-EF. São Paulo: Casa do Psicólogo, 2010.

PINQUART, M.; SHEN, Y. Behavior problems in children and adolescents with chronic physical illness: a meta-analysis. Journal of Pediatric Paychology, v. 36, n. 9, p. 003-1016, 2011.

PINQUART, M.; TEUBERT, D. Academic, physical and social functioning of children and adolescents with chronic physical illness: a meta analysis. Journal of Pediatric Paychology, V. 37, N. 4, p. 376-38, 2011.

PRATES, K. C. R.; LIMA, R. F.; CIASCA, S. M. Estratégias de aprendizagem e suas relações com o desempenho escolar em crianças do Ensino Fundamental I. Revista Psicopedagogia, v. 33, n. 100, p. 19-27, 2016.

RIBEIRO, C. R.; JUNIOR, A. A. P. A representação social da criança hospitalizada: um estudo por meio do procedimento de desenho-estória com tema. Rev. SBPH [online]; v.12, n.1, p. 31-56, 2009.

SANCHEZ, M. L. M.; EBELING, V. L. N. Internação infantil e sintomas depressivos: intervenção psicológica. Rev. SBPH, v. 14, n. 1, p. 186-199, 2011. Disponível em <http://pepsic.bvsalud.org/scielo.php?script=sci_arttext\&pid=S1516-08582011000100011\&lng=pt\&nrm=iso >.

SILVEIRA, K. A; , LIMA, V. L.; PAULA, K. M. P. Estresse, dor e enfrentamento em crianças hospitalizadas: análise de relações com o estresse do familiar. Revista da SBPH, v. 21, n. 2, p. 5-21, 2018. http://pepsic.bvsalud.org/scielo. 
php?script=sci_arttext\&pid=S1516-08582018000200002\&lng=pt\&nrm=iso

SILVEIRA, K. A.; Paula, K. M. P.; Enumo, S. R. F. Estresse Relacionado à Hospitalização Pediátrica e Intervenções Possíveis: Análise da Literatura Brasileira. Temas Em Psicologia, v. 27, n. 2, p. 443-458, 2019. https://doi.org/10.9788/ TP2019.2-11

SIMONTETTI, A. Manual de Psicologia Hospitalar: o mapa da doença. São Paulo: Casa do Psicólogo, 2004.

XAVIER, T. G. M. Escolarização de crianças e adolescentes hospitalizados: do direito à realidade. Dissertação (Mestrado em Enfermagem). Programa de Pós-Graduação em Enfermagem. Universidade Federal da Paraíba. João Pessoa: UFPB, 2012. 